\title{
CLINICOPATHOLOGICAL ANALYSIS OF POLYPOID LESIONS OF CERVIX
}

Sidhalingreddy, Shilpa Biradar, Akhila, V. D. Domble,
1. Assistant Professor. Department of Pathology, SN Medical College, Navanagar. Bagalkot.
2. Assistant Professor. Department of Pathology, SN Medical College, Navanagar. Bagalkot.
3. PG Cum Tutor. Department of Pathology, SN Medical College, Navanagar. Bagalkot.
4. Professor \& Head. Department of Pathology, SN Medical College, Navanagar. Bagalkot.

\section{CORRESPONDING AUTHOR:}

Dr. Sidhalingreddy,

Assistant Professor,

Department of pathology

S. N. Medical College,

Navanagar, Bagalkot- 587102.

E-mail: drssreddybenoor@gmail.com

ABSTRACT: BACKGROUND: Polypoidal lesions of cervix show various morphological spectrum ranging from inflammatory to neoplastic lesions. In addition to endocervical polyps there are many other polypoidal lesions that appear clinically like endocervical polyps. They are often secondarily inflamed or ulcerated and contain foci of squamous metaplasia. AIM: To study clinicopathological aspects of cervical polypoid lesions. OBJECTIVES: To study the histopathological features of cervical polypoid lesions and to assess their associated clinical features and age wise incidence. MATERIALS AND METHODS: All specimens mentioned as cervical polyp based on the clinical appearance were included in the study. All such polyps from cervix/cervical os were removed and sent for histopathological examination to our department. In some cases we got complete polyp and in some fragments of polyp. All these Specimens obtained with relevant clinical data were formalin fixed and processed with standard procedures. Sections were stained with routine Haematoxylin and eosin stain. We studied various histopathological features and analysed for associated clinical features. RESULTS: During the study period we got 22 cases of cervical polyps. They are common between 20$50 y r s$. Common associated clinical features were history of bleeding per vagina or white discharge per vagina. The most common type of polypoidal lesion in this study was classical Endocervical polyp(13). Most of them showed cystic changes and nearly half of endocervical polyps showed squamous metaplasia . Other polypoidal lesions we encountered in this study were Leiomyoma (3), Adenomatous polyp (1), Adenoleiomyoma (1) and Nabothian cyst (2).

CONCLUSION: It appears that the entities such as Polypoidal endocervicitis , Endocervical polyp and Fibroepithelial polyp belong to spectrum of a single entity in the pathogenesis process with Polypoidal endocervicitis as initial lesion, Endocervical polyp as intermediate lesion and Fibroepithelial polyp as a last stage in the pathogenesis process of same entity. They need to be removed in symptomatic cases and in cases where there is high probability of harboring malignant foci. Regular followup can be done in other cases. Further large studies needs to be conducted to evaluate various aspects of endocervical polyps.

KEY WORDS: cervix, polypoid, histopathology.

INTRODUCTION: Cervical polyps are polypoidal growths from the cervix often look like bulbs on thin stems. They are thought to result from chronic inflammation. Cervical polyps are usually benign and can occur alone or in groups. Most polyps are small, about 1- 2 centimeters long [1,2]. 
In addition to endocervical polyps there are many other polypoidal lesions that appear clinically like endocervical polyps. Because rare types of polypoidal malignancy can look like endocervical polyps and endocervical polyps rarely harbour malignant foci all polyps should be removed and examined microscopically[3,4]. These polyps are sometimes referred as mucous polyps consisting of inflammatory cells. Many non-neoplastic and neoplastic lesions also clinically look like polyps. These include Adenomyoma, Embryonal rhabdomyosarcoma, Leiomyoma, and even lesions like Schwannoma, Nabothian cyst, Decidualized stroma, Plasmacytoma, Villoglandular papillary adenocarcinoma, Adenosarcoma, Non-hodgkin's lymphoma, Aggressive angiomyxoma, Gliosis of cervix etc.[5-13]. These may be polyps from ectocervix or polyps from endocervix which may be labeled as ectocervical polyps and endocervical polyps respectively. The most common type of endocervical polyp consists of a polypoid growth surfaced by benign mucinous epithelium and containing numerous crypts, some cystic, lined by similar epithelium. These polyps are often secondarily inflamed or ulcerated and usually contain foci of squamous metaplasia. Other less common variants include polyps composed predominantly of fibrous or vascular tissue. The latter variant often presents with bleeding. Polyps arising from the upper part of the canal or the lower uterine segment are often composed of a mixture of endocervical and endometrial epithelium and are thus designated as mixed endocervical-endometrial polyps $[1,3,4]$. This study was done to assess clinicopathological aspect of polypoid lesions of cervix in this region.

MATERIALS AND METHODS: This study was carried out in the department of pathology for a period of three years from September 2009 to August 2012. All specimens mentioned as cervical polyp on gross or per speculum examination were included in the study. Those hysterectomy specimens which appeared malignant on gross examination even with polypoidal presentation were excluded from the study. Those cervical polyps with clinical diagnosis of suspected malignancy were also excluded from the study. They were named as cervical polyp based on the clinical appearance on examination. All such polyps from cervix/cervical os were removed and sent for histopathological examination to our department. In some cases we got complete polyp and in some fragments of polyp. All these Specimens obtained with relevant clinical data were formalin fixed and processed with standard procedures. Sections were stained with routine Haematoxylin and eosin stain. We studied sections with respect to various histopathological features and analysed for associated clinical features.

RESULTS: During the study period we got 22 cases of cervical polyps. They are common between 20-50yrs. No cases were seen before 20yrs. Only one case seen after 50yrs. Common associated clinical features were history of bleeding per vagina or white discharge per vagina. Few presented with history of mass per vagina, menstrual irregularities like menorrhagia, polymenorrhagia and abdominal pain. In two cases no associated clinical features were seen. Polyps were detected during per speculum examination or gross examination. Few presented with more than one symptom [Table.1].

The most common type of polypoidal lesion in this study was classical Endocervical polyp(13) [Table 2]. By definition these are the lesions which consisted histological features like loose edematous stroma, few endocervical glands and chronic inflammatory cells consisting predominantly lymphocytes and few plasma cells (Figure 1). These in few cases may show little fibrosis. A variant with predominant fibrosis and epithelial lining were labeled as Fibroepithelial polyp (Figure 2). We encountered two such cases in this study. Few Endocervical 
polyps showed acute inflammatory cells consisting of neutrophils especially at edge of the polyp. Few lesions showed vascular congestion (Figure 3). Most of them showed cystic changes (Figure 4) and nearly half of Endocervical polyps showed squamous metaplasia (Figure 5 and 6). Chronic inflammatory cell infiltration was scant to moderate in all Endocervical polyps. Non of the Endocervical polyps showed granulomatous reaction or dysplastic changes or foci of malignancy. Other polypoidal lesions we encountered in this study were Leiomyoma (3), Adenomatous polyp (1), Adenoleiomyoma (1) and Nabothian cyst(2). Scant lymphocytic infiltration was common in all these lesions. Those with only whorling bundles of plump smooth muscle component were put in leiomyoma category (Figure 7). Adenomatous polyps showed glandular component. Adenoleiomyoma showed both plump smooth muscle cells in whorling pattern and glandular component. Nabothian cysts were cystic structures which showed atrophied lining epithelium and cystic fluid inside (Figure 8).

DISCUSSION: Polypoid lesions of cervix can be neoplastic or non-neoplastic in nature. Those polyps of inflammatory nature are usually referred as endocervical polyps which show loose edematous stroma, endocervical glands usually with cystic change and chronic inflammatory cell infiltrate consisting of lymphocytes and plasma cells. Fibrosis may or may not be seen. The so called polypoidal endocervicitis shows similar features. Fibroepithelial polyps show predominant fibrous component. This study showed that Endocervical polyps are seen commonly between 20-50years age group and Fibroepithelial polyps in 40-50years age group. It appears that the entities such as Polypoidal endocervicitis, Endocervical polyp and Fibroepithelial polyp belong to spectrum of a single entity in the pathogenesis process with Polypoidal endocervicitis as initial lesion, Endocervical polyp as intermediate lesion and Fibroepithelial polyp as a last stage in the pathogenesis process of same entity. There are numerous categories of lesions ranging inflammatory to benign to malignancy which may present clinically as polypoidal lesions and many case reports have been published [5-13]. The risk of cervical polyps harboring malignant foci is one possibility and is of very minimal chance. Very little literature available indicates $0.0-1.7 \%$ malignant change. But one study involving 1366 cases of cervical polyps showed no malignant change in cervical polyps [14]. This study even questioned need of removing such polyps unless they are symptomatic. Similar to this study we also encountered few cases of asymptomatic cervical polyps. Pattern of clinical presentation is also in consistent with previous literature. Few studies recommend their surgical removal both symptomatic and asymptomatic polyps in view of their varying morphologic spectrum and possibility of recurrence. Studies suggest to remove them to exclude the possibility of malignancies presenting as polyps and rare polyps containing dysplastic/malignant foci. Endocervical polyps are the most common type of polypoid lesion we found and is in consistent with previous findings. Histologic changes like squamous metaplasia and cystic changes are in consistent with available literature ${ }^{[1-4,14]}$.

CONCLUSION: It appears that the entities such as Polypoidal endocervicitis, Endocervical polyp, and Fibroepithelial polyp belong to spectrum of a single entity in the pathogenesis process with Polypoidal endocervicitis as initial lesion, Endocervical polyp as intermediate lesion and Fibroepithelial polyp as a last stage in the pathogenesis process of same entity. They need to be removed in symptomatic cases and in cases where there is high probability of harboring malignant foci. Regular followup can be done in other cases. Further large studies needs to be conducted to evaluate various aspects of endocervical polyps. 


\section{REFERENCES:}

1. NHS foundation trust. Doncaster and Bassetlaw hospitals [Internet]. Cervical polyps [leaflet];2012.

Available

from:http://www.dbh.nhs.uk/library/patient_information_leaflets/WPR22220cervical\%20polyps.pdf.

2. Juan Rosai. Uterus cervix. In: Rosai and ackerman's surgical pathology. 9th ed. Newdelhi: Elsevier ;2004.p.1523-1551.

3. Seema anushka tirlapur, Adewale adeyemo, Neil o'gorman, Dan selo-ojeme. Clinico pathological study of cervical polyps. Archieves of gynecology and obstetrics. November 2010;282(5):535-538.

4. Keith V Nance, Endocervical polyps. REX Laboratory bulletin. 2009;150:1-2. Available from:https://www.rexhealth.com/workfiles/services/lab/Bull05-2009.pdf

5. Hino A, Seki, Sano N. Adenosarcoma of the uterine cervix presenting as a cervical polyp. Pathology international. 1998;48(8):649-652.

6. Kalpana A Bothale. A rare presentation of aggressive angiomyxoma as a cervical polyp. 2012;1(4):239. Available from:http//dx.doi.org/10.4172/scientificreports.239.

7. Pemasiri upali telisinghe, Ghazala kafeel, Hla oo. Gliosis of the cervix. Brunei int med j. 2011;7(3): 173-176.

8. Azamsadat mousavi, Setare akhavan. Sarcoma botryoides (embryonal rhabdomyosarcoma) of the uterine cervix in sisters. J gynecol oncol December. 2010;21(4):273-275.

9. Vasudev DS, Kaler AK. Non-hodgkin's lymphoma of the uterine cervix. Online $\mathrm{j}$ health allied scs. 2012;11(1):13. Available from: http://www.ojhas.org/issue41/2012-113.html.

10. Louis P Dehner, Jason A Jarzembowski and D Ashley hill. Embryonal rhabdomyosarcoma of the uterine cervix: a report of 14 cases and a discussion of its unusual clinicopathological associations. Modern pathology. 2012;25:602-614.

11. Edgar G. Fischer. Primary plasmacytoma arising in an endocervical polyp with detection of neoplastic cells on papanicolaou test. A Case Report and Review of the Literature . Arch pathol lab med. 2003;127: 28-31.

12. Okogbo fo, Ezechi oc, Loto om, Ezeobi pm. Uterine leiomyomata in south western nigeria: A clinical study of presentations and management outcome. African health sciences. 2011;11(2):271-278.

13. P.S.Srilatha, A. Roy. Villoglandular papillary adenocarcinoma of cervix associated with cervical intraepithelial neoplasia : a case report and review of literature. 2007;50(4):819-21.

14. Mackenzie i, Naish C., Rees C, Manek S. Why remove all cervical polyps and examine them histologically? BJOG. 2009;116:1127-1129. 
Table.1. Cervical polypoid lesions.- Age distribution and their associated clinical features

\begin{tabular}{|c|c|c|c|c|c|}
\hline \multirow[t]{2}{*}{ Associated clinical features } & \multicolumn{4}{|c|}{ Age in years } & \multirow[t]{2}{*}{ Total } \\
\hline & $21-30$ & $31-40$ & $41-50$ & $51-60$ & \\
\hline Bleeding per vagina & 1 & 3 & 4 & & 8 \\
\hline White discharge per vagina & 3 & 2 & 1 & & 6 \\
\hline Mass per vagina & & & 3 & & 3 \\
\hline Abdominal pain & & 2 & & & 2 \\
\hline Irregular menstrual cycles & & 2 & 1 & & 3 \\
\hline No associated clinical features & & & 1 & 1 & 2 \\
\hline Total & 4 & 9 & 10 & 1 & \\
\hline
\end{tabular}

Table.2. Histopathological spectrum of cervical polypoid lesions.

\begin{tabular}{|l|l|l|l|l|l|}
\hline \multirow{2}{*}{ Histologic type of polyps } & \multicolumn{3}{|l|}{ Age (in years) } & \multirow{2}{*}{ Total } \\
\cline { 2 - 5 } & $21-30$ & $31-40$ & $41-50$ & $51-60$ & \\
\hline Endocervical polyp & 3 & 5 & 5 & & 13 \\
\hline Leiomyomatous polyp & & 2 & 1 & & 3 \\
\hline Adenomatous polyp & & 1 & & & 1 \\
\hline Fibroepithelial polyp & & & 2 & & 2 \\
\hline $\begin{array}{l}\text { Adeno leiomyomatous } \\
\text { polyp }\end{array}$ & 1 & & & & 1 \\
\hline Nabothian cyst & & & & & \\
\hline Total & 4 & 8 & 9 & 1 & 22 \\
\hline
\end{tabular}




\section{ORIGINAL ARTICLE}

Figure.1.Lymphocytes in endocervical polyp. 40x (H\&E).

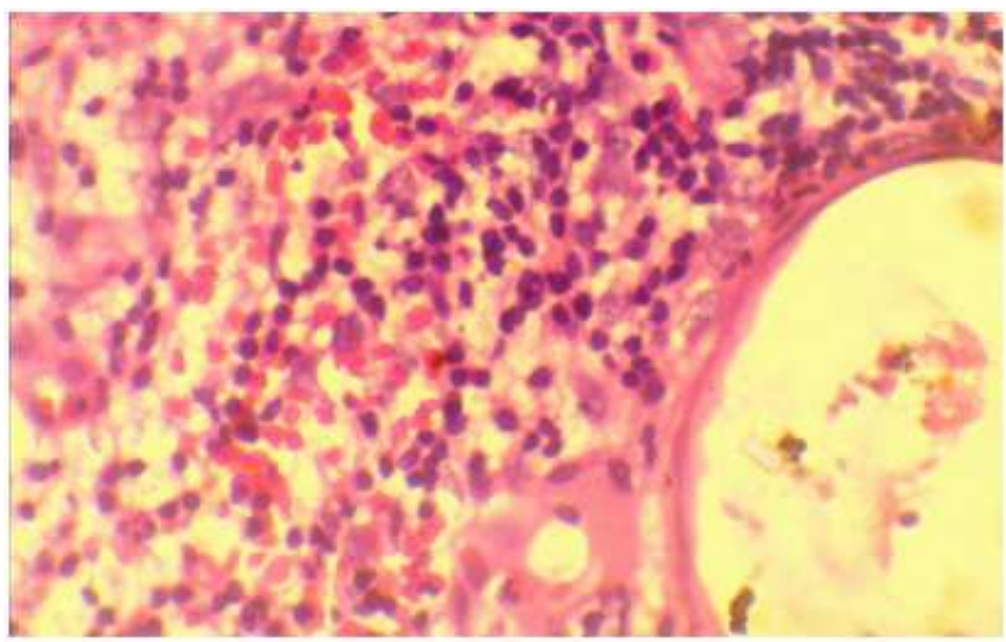

Figure. 2. Fibroepithelial polyp. 10x (H\&E)

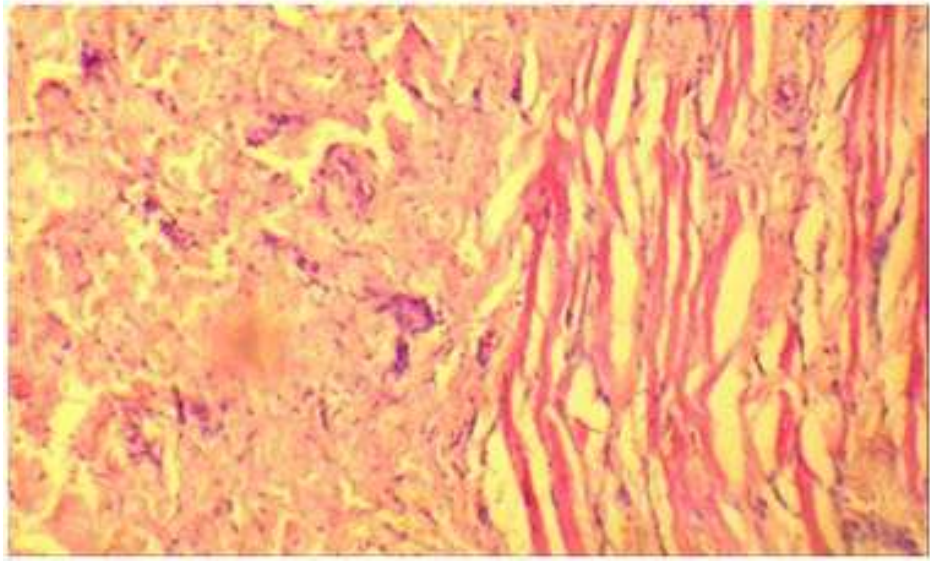

Figure 3. Vascular congestion in endocervical polyp. 10x(H\&E)

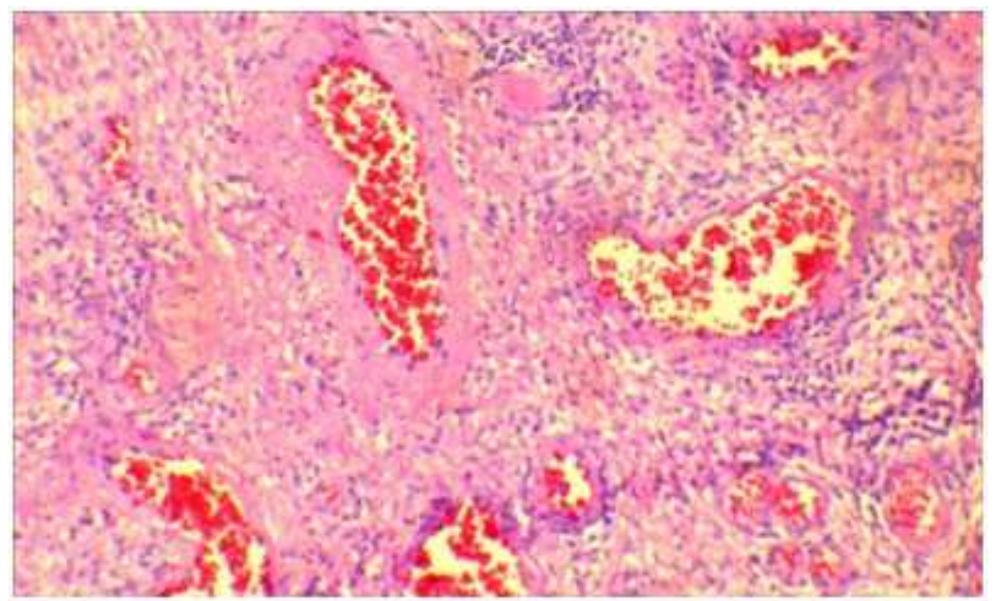


Figure 4. Endocervical polyp with cystic changes. 5x(H\&E)

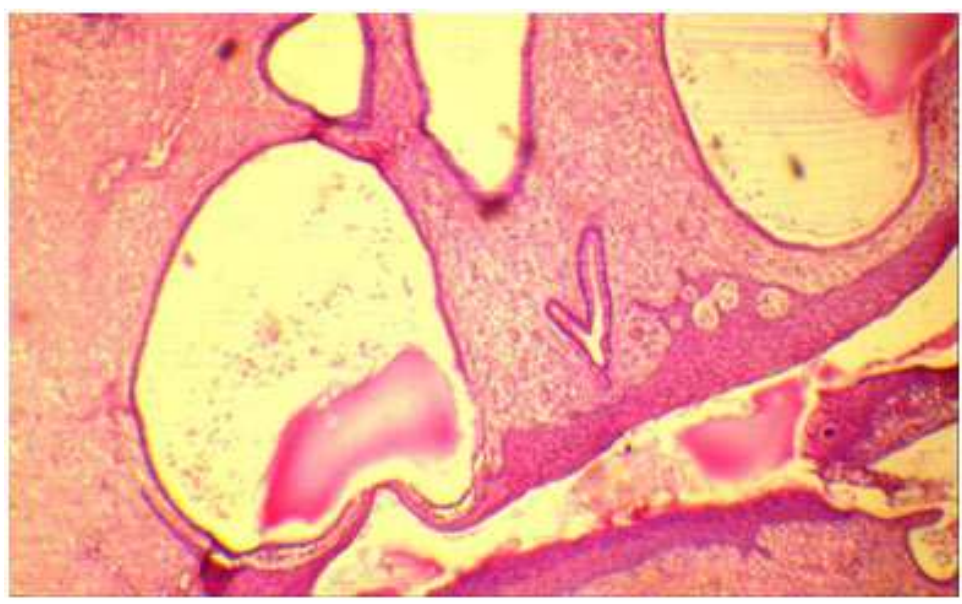

Figure 5. Endocervical polyp showing squamous metaplasia. 10x (H\&E).

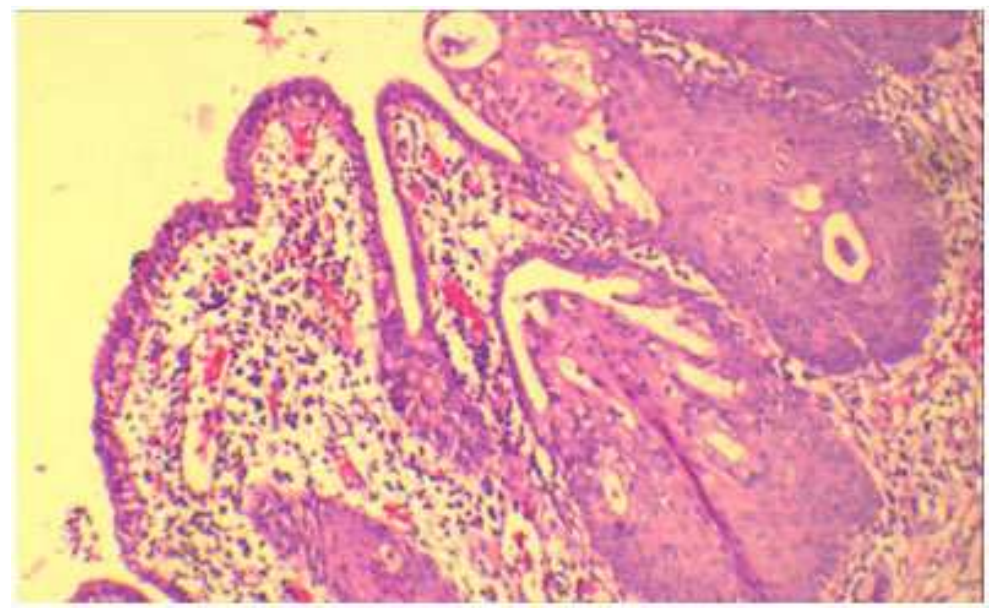

Figure 6. Squamous metaplasia of endocervical gland. 10x (H\&E)

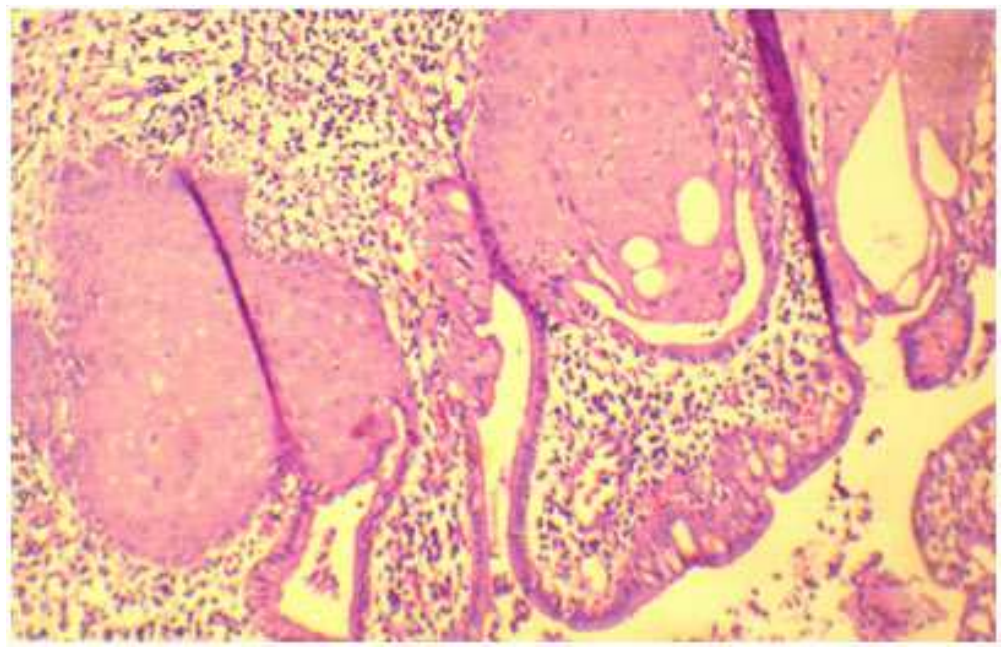




\section{ORIGINAL ARTICLE}

Figure 7. Leiomyoma presenting as cervical polyp. 10x (H\&E).

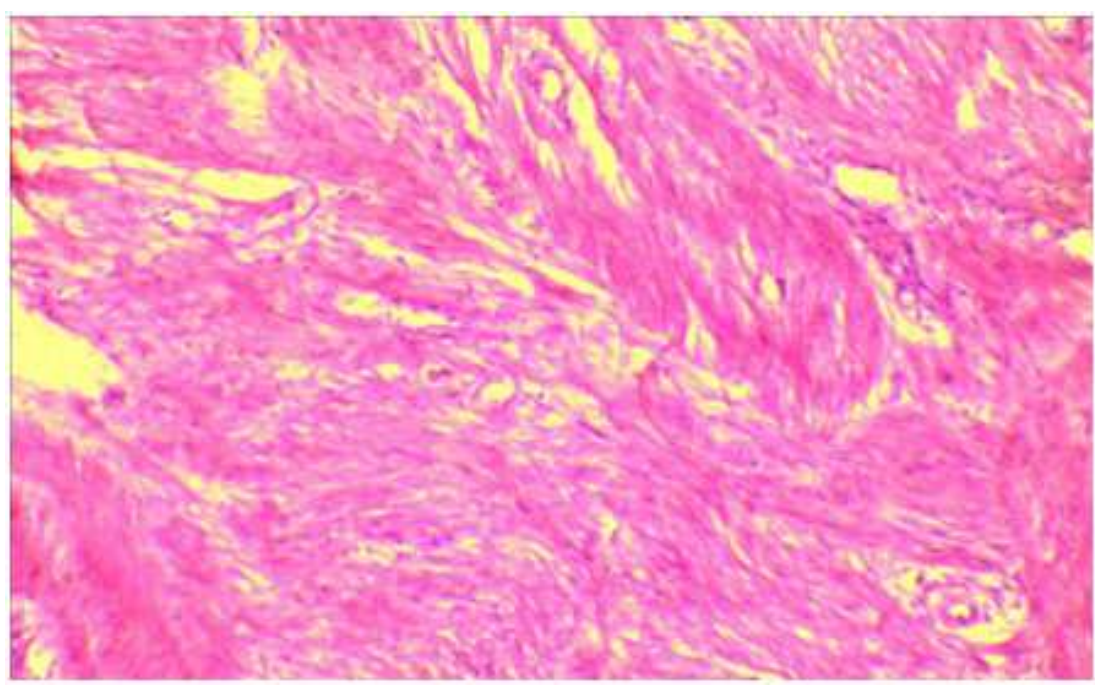

Figure 8. Nabothian cyst. 4x (H\&E).

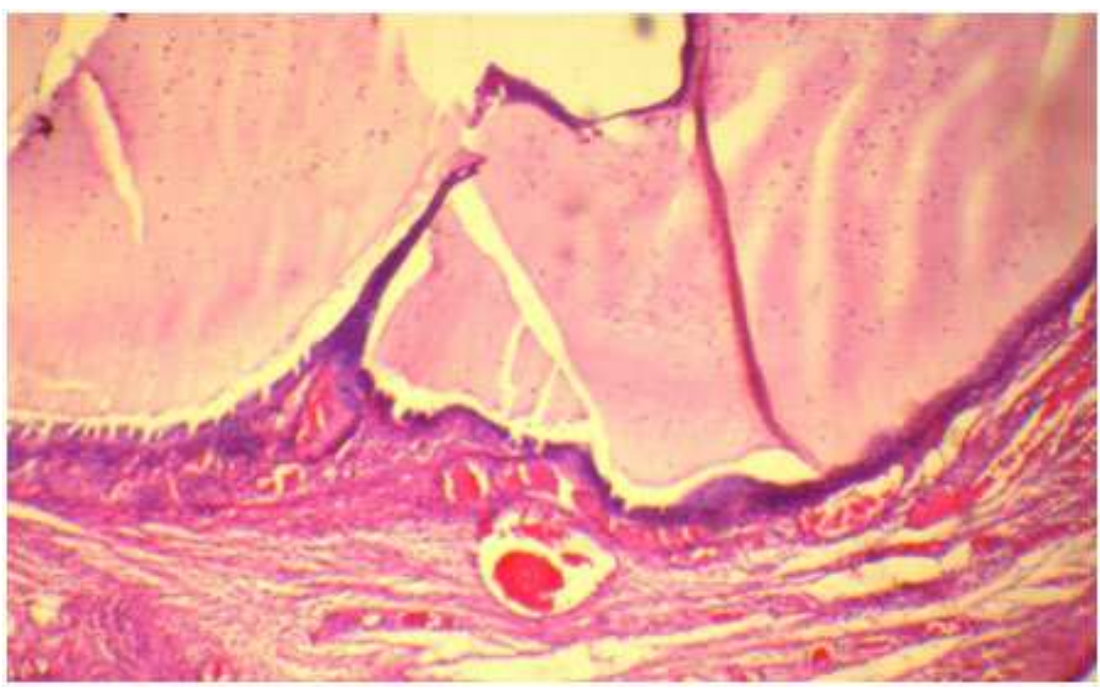

